\title{
Molecular and Cellular Organization of the Taste System in the Drosophila Larva
}

\author{
Jae Young Kwon, ${ }^{1,2}$ Anupama Dahanukar, ${ }^{1,3}$ Linnea A. Weiss, ${ }^{1}$ and John R. Carlson ${ }^{1}$ \\ ${ }^{1}$ Department of Molecular, Cellular, and Developmental Biology, Yale University, New Haven, Connecticut 06520-8103, ${ }^{2}$ Department of Biological Sciences, \\ Sungkyunkwan University, Suwon 440-746, Republic of Korea, and ${ }^{3}$ Department of Entomology, University of California, Riverside, Riverside, California \\ 92521
}

We examine the molecular and cellular basis of taste perception in the Drosophila larva through a comprehensive analysis of the expression patterns of all 68 Gustatory receptors (Grs). Gr-GAL4 lines representing each Gr are examined, and 39 show expression in taste organs of the larval head, including the terminal organ (T0), the dorsal organ (D0), and the pharyngeal organs. A receptor-to-neuron map is constructed. The map defines 10 neurons of the TO and DO, and it identifies 28 receptors that map to them. Each of these neurons expresses a unique subset of Gr-GAL4 drivers, except for two neurons that express the same complement. All of these neurons express at least two drivers, and one neuron expresses 17. Many of the receptors map to only one of these cells, but some map to as many as six. Conspicuously absent from the roster of Gr-GAL4 drivers expressed in larvae are those of the sugar receptor subfamily. Coexpression analysis suggests that most larval Grs act in bitter response and that there are distinct bitter-sensing neurons. A comprehensive analysis of central projections confirms that sensory information collected from different regions (e.g., the tip of the head vs the pharynx) is processed in different regions of the suboesophageal ganglion, the primary taste center of the CNS. Together, the results provide an extensive view of the molecular and cellular organization of the larval taste system.

\section{Introduction}

The Drosophila larva provides a numerically simple and genetically tractable model system in which to study the molecular and cellular basis of taste (Stocker, 2008). Three major external chemosensory organs lie on the anterior tip of the larval head: the dorsal organ (DO), the terminal organ (TO), and the ventral organ (VO) (see Fig. 1A). The multiporous dome of the DO has olfactory function (Oppliger et al., 2000), while six peripheral DO sensilla have terminal pores indicative of gustatory function, as do most sensilla in the TO and VO (Stocker, 1994). The cell bodies of neurons that innervate the DO lie in the DO ganglion (DOG). Neurons that innervate the TO fall into two groups, the dorsolateral group, which has cell bodies in the DOG, and the distal group, which has cell bodies in the TO ganglion (TOG) (Stocker, 1994; Vosshall and Stocker, 2007). The VO ganglion (VOG) contains the cell bodies of gustatory neurons innervating the VO. Three chemosensory organs lie in the pharynx: the dorsal, ventral, and posterior pharyngeal sense organs (DPS, VPS, and PPS, respectively) (see Fig. 1A) (Gendre et al., 2004).

\footnotetext{
Received July 2, 2011; revised Aug. 20, 2011; accepted Aug. 25, 2011.

Author contributions: J.Y.K. and J.R.C. designed research; J.Y.K., A.D., and L.A.W. performed research; J.Y.K. analyzed data; J.Y.K. and J.R.C. wrote the paper.

This work was supported by NIH grants (J.R.C.), the Basic Research Promotion Fund (National Research Foundation of Korea Grant 2009-0075727), and the (onversing Research (enter Program through the National Research Foundation of Korea funded by Ministry of Education, Science and Technology Grant 2011 K000696 (J.Y.K.). We thank A. Hofbauer, K. Scott, and H. Amrein for reagents and flies, and members of the Carlson Laboratory for comments on this manuscript.

Correspondence should be addressed to John R. Carlson at the above address. E-mail: john.carlson@yale.edu. DOI:10.1523/JNEUROSCI.3363-11.2011

Copyright $\odot 2011$ the authors $\quad 0270-6474 / 11 / 3115300-10 \$ 15.00 / 0$
}

Neurons in the DOG project to the brain via the antennal nerve, and neurons from the TOG and VOG project to the brain via the maxillary nerve (Stocker, 1994; Python and Stocker, 2002; Vosshall and Stocker, 2007). Neurons in the DPS and PPS extend to the brain via the labral nerve, while the labial nerve carries projections from the VPS. The major taste center in the larval brain is the subesophageal ganglion (SOG) (Colomb et al., 2007; Vosshall and Stocker, 2007).

The Gustatory receptor (Gr) gene family contains 60 members that encode 68 proteins through alternative splicing (Clyne et al., 2000; Dunipace et al., 2001; Scott et al., 2001; Robertson et al., 2003). Gr5a and two closely related receptors encode sugar receptors (Dahanukar et al., 2007; Slone et al., 2007; Jiao et al., 2008); Gr33a, Gr32a, Gr66a, and Gr93a mutants are defective in bitter reception (Moon et al., 2006, 2009; Lee et al., 2009, 2010). In the adult, Gr5a and Gr66a are expressed in distinct subsets of gustatory receptor neurons to mediate sweet or bitter taste and acceptance or avoidance behavior (Thorne et al., 2004; Wang et al., 2004; Marella et al., 2006).

Among the 68 gustatory receptors, expression patterns of only $15 \mathrm{Gr}$ genes have been examined in detail in the larva, using the GAL4-UAS system (Colomb et al., 2007; Thorne and Amrein, 2008). Ten other Gr-GAL4 lines did not show larval expression. A comprehensive analysis, however, has not been performed, and the complete larval repertoire has not even been identified.

Here, we systematically examine the expression of all Gr genes in the Drosophila larva and the projections of expressing neurons in the CNS. We define 10 gustatory neurons and provide a receptor-toneuron map. The results suggest that receptors are expressed combinatorially and that most detect bitter compounds. 


\section{Materials and Methods}

Drosophila stocks and transgenes. Flies were cultured on standard cornmeal agar medium at room temperature $\left(23 \pm 2^{\circ} \mathrm{C}\right)$. $w$; UAS-mCD8-GFP was used as the GFP reporter (Lee and Luo, 1999).

The 5' upstream regions used to construct the Gr-GAL4 transgenes are described by Weiss et al. (2011). Briefly, 59 Gr-GAL4 transgenes were constructed and $8 \mathrm{Gr}$-GAL4 transgenes were kindly provided by H. Amrein (Texas A\&M University, College Station, TX) and K. Scott (University of California, Berkeley, Berkeley, CA), for a total of $67 \mathrm{Gr}$-GAL4 transgenes. To construct the Orco-RFP transgene, the Orco promoter (8.3 $\mathrm{kb})$ was cloned into the RFP-IRES-RFP-IRES-RFP expression vector used in the study by Dahanukar et al. (2007), and injected into $w^{1118}$. Orco-RFP expression completely overlaps with the expression of the 21 larval olfactory receptor neurons (ORNs) in Orco-GAL4; UAS-mCD8GFP flies (data not shown).

Quantitative analysis of expression and selection of representative lines. Different insertions of an individual promoter-GAL4 transgene may exhibit variations in expression pattern due to positional effects. In an effort to identify Gr-GAL4 lines that show expression patterns faithful to those of the endogenous $G r$ genes, we examined as many independent lines as possible for a given Gr-GAL4 transgene. For each independent Gr-GAL4 line, we crossed Gr-GAL4 homozygotes and flies with UAS$m C D 8$-GFP homozygous on both chromosomes 2 and 3 , and scored GFP reporter expression in at least 10 progeny of either sex at the third-instar larval stage. An effort was made to select larvae from within the culture medium, as opposed to older, third-instar larvae at the wandering stage. These progeny were heterozygous for the Gr-GAL4 driver and contained two copies of the UAS- $m C D 8$-GFP reporter.

To quantitate expression of each Gr-GAL4 line, we counted the number of GFP-labeled cells on both the left and right sides of all larvae and calculated the average number of labeled cell pairs. For example, if we examined 10 larvae of a particular Gr-GAL4 line and observed that seven animals expressed GFP in one TO-distal neuron on each side $(7 \times 2=$ $14)$, two animals expressed GFP in only one neuron on one side $(2 \times 1=$ $2)$, and one animal did not show GFP expression $(1 \times 0=0)$, the maximum number of labeled TO-distal neurons is 1 (Table 1 ), and the average expression level is $(14+2+0) / 20=0.8$ (data not shown). No Gr-GAL4 drivers showed labeling in a pattern indicative of left/right asymmetry.

For each Gr-GAL4 transgene, one pattern was observed in the majority of independent lines. Among lines showing this most common pattern, we selected as the representative line the one that showed the most penetrant expression, based on the calculations described above.

To examine whether two different Gr-GAL4 transgenes labeled the same or different neurons, we crossed the representative lines for each $G r-G A L 4$ transgene with a stock carrying UAS-mCD8-GFP to score progeny with one copy of each Gr-GAL4 transgene and two copies of $m C D 8$ GFP. The number of neuron pairs expressing GFP was determined by the same method described above. The number of labeled neurons was quantified in at least 10 animals for each double-driver combination. We note that expression of the Gr66a-RFP transgene that was used to analyze coexpression in the adult by Weiss et al. (2011) was too weak to be useful in the larva and that it is now possible to alleviate position effects via use of the phiC31 system (Groth et al., 2004; Fish et al., 2007).

Fluorescence imaging and immunostaining. GFP and RFP expression were imaged directly in analysis of peripheral expression in the larval head. Larval heads were removed from the body with microscissors and incubated in 50\% glycerol in PBS for $1 \mathrm{~h}$ before direct observation of fluorescence.

Larval brains were dissected and immunostained as previously described (Python and Stocker, 2002). Anti-GFP (rabbit polyclonal; Invitrogen; 1:1000 dilution) was used to enhance the GFP signals of UAS$m C D 8-G F P$. UAS- $m C D 8-G F P$ allows visualization of the general morphology of GAL4-expressing cells. Synaptic areas were visualized with mouse monoclonal antibody nc82 (1:100) (a gift from Dr. Alois Hofbauer, University of Regensburg, Regensburg, Germany). nc82 recognizes the ELKS/CAST/ERC family protein Bruchpilot, which localizes to presynaptic active zones (Laissue et al., 1999; Wagh et al., 2006). The
Table 1. Summary of Gr expression patterns in the TO, DO, and pharyngeal organs

\begin{tabular}{|c|c|c|c|c|c|c|c|}
\hline & \multirow[b]{2}{*}{ Lines $^{a}$} & \multicolumn{2}{|l|}{ T0 } & \multirow[b]{2}{*}{ DO } & \multicolumn{3}{|c|}{ Pharyngeal organs } \\
\hline & & Distal & Dorsolateral & & DPS & VPS & PPS \\
\hline Gr2a & $7 / 8$ & 2 & - & 2 & 1 & - & - \\
\hline Gr5a & $7 / 7$ & - & - & - & - & - & - \\
\hline Gr8a & $5 / 5$ & - & - & - & - & - & - \\
\hline Gr9a & $7 / 10$ & 1 & - & - & - & - & - \\
\hline Gr10a & $7 / 8$ & - & 1 & - & - & - & - \\
\hline Gr10b & $1 / 1$ & - & - & - & - & - & - \\
\hline Gr27a & $1 / 1^{b}$ & 1 & - & - & - & - & - \\
\hline Gr22a & 9/11 & 1 & - & - & - & - & - \\
\hline$G r 22 b$ & $14 / 15$ & - & - & - & 1 & - & 1 \\
\hline Gr22C & $1 / 1^{b}$ & - & - & - & - & - & - \\
\hline Gr22d & $1 / 1$ & - & - & - & 1 & - & - \\
\hline Gr22e & $6 / 7$ & 1 & - & - & 1 & - & - \\
\hline Gr22f & $6 / 6$ & - & - & - & - & - & - \\
\hline Gr23a & $6 / 7$ & - & - & - & 1 & - & - \\
\hline Gr28a & $3 / 3^{c}$ & 2 & - & 2 & - & 2 & 2 \\
\hline Gr28b.a & $15 / 15$ & 1 & - & - & 1 & - & - \\
\hline$G r 28 b . b$ & $9 / 12$ & - & - & - & - & - & - \\
\hline Gr28b.c & $4 / 4$ & - & - & - & - & - & - \\
\hline Gr28b.d & $1 / 1^{c}$ & - & - & - & - & - & - \\
\hline Gr28b.e & $1 / 1^{b}$ & 1 & - & - & - & - & - \\
\hline Gr32a & $2 / 2$ & 1 & 1 & - & 2 & - & 2 \\
\hline Gr33a & $8 / 12$ & 4 & 2 & - & 2 & 2 & 2 \\
\hline Gr36a & $7 / 8$ & - & - & - & - & - & - \\
\hline Gr36b & $8 / 8$ & 1 & - & - & - & - & - \\
\hline Gr36c & $5 / 5$ & 1 & - & - & - & - & - \\
\hline Gr39a.a & $1 / 1$ & 2 & - & - & 1 & - & 2 \\
\hline Gr39a.b & $3 / 3$ & 2 & - & - & 1 & - & - \\
\hline Gr39a.c & $1 / 1$ & - & - & - & - & - & - \\
\hline Gr39a.d & $1 / 1$ & - & - & - & - & - & 2 \\
\hline Gr39b & $5 / 5$ & - & - & - & 1 & - & 2 \\
\hline Gr43a & $17 / 23$ & - & - & - & 1 & - & - \\
\hline Gr47a & $1 / 1^{b}$ & - & - & - & - & - & - \\
\hline Gr47b & $5 / 7$ & 1 & - & - & - & - & - \\
\hline Gr57a & $7 / 7$ & 2 & - & - & 1 & - & - \\
\hline Gr58a & $15 / 15$ & - & - & - & - & - & - \\
\hline Gr58b & $9 / 10$ & 1 & - & - & 1 & - & - \\
\hline Gr58c & $6 / 6$ & - & - & - & - & - & - \\
\hline Gr59a & $11 / 15$ & 1 & - & - & - & - & - \\
\hline Gr59b & $1 / 1^{c}$ & - & - & - & - & - & - \\
\hline Gr59c & $12 / 13$ & 1 & - & - & - & - & - \\
\hline Gr59d & $3 / 3$ & 3 & - & - & 1 & - & - \\
\hline Gr59e & $13 / 16$ & 1 & - & - & - & - & - \\
\hline Gr59f & $6 / 8$ & 1 & - & - & - & - & - \\
\hline Gr61a & $7 / 10$ & - & - & - & - & - & - \\
\hline Gr63a & $5 / 13$ & 1 & - & - & - & - & - \\
\hline Gr64a & $9 / 9$ & - & - & - & - & - & - \\
\hline Gr64b & $8 / 9$ & - & - & - & - & - & - \\
\hline Gr64c & $4 / 4$ & - & - & - & - & - & - \\
\hline Gr64d & $7 / 8$ & - & - & - & - & - & - \\
\hline Gr64e & $3 / 3$ & - & - & - & - & - & - \\
\hline Gr64f & $4 / 5$ & - & - & - & - & - & - \\
\hline Gr66a & $6 / 6$ & 4 & 2 & - & 2 & 2 & 2 \\
\hline Gr68a & $3 / 3^{c}$ & - & - & - & - & 2 & - \\
\hline Gr77a & $9 / 14$ & - & - & - & 1 & - & - \\
\hline Gr85a & $15 / 19$ & - & - & - & - & - & - \\
\hline Gr89a & $10 / 14$ & - & - & - & - & - & - \\
\hline Gr92a & $12 / 12$ & - & - & - & - & - & - \\
\hline Gr93a & $12 / 14$ & - & - & - & 1 & - & - \\
\hline Gr93b & $10 / 10$ & 1 & - & - & 1 & - & - \\
\hline Gr93c & $8 / 8$ & - & - & - & - & - & 2 \\
\hline Gr93d & 9/14 & - & - & - & - & - & 2 \\
\hline Gr94a & $12 / 12$ & 1 & - & - & - & - & - \\
\hline Gr97a & $4 / 4$ & 1 & - & - & - & - & - \\
\hline Gr98a & $1 / 1$ & - & - & - & - & - & - \\
\hline Gr98b & $2 / 2$ & - & - & - & - & - & - \\
\hline Gr98c & $3 / 4$ & - & - & - & - & - & - \\
\hline Gr98d & $15 / 15$ & - & - & - & - & - & - \\
\hline
\end{tabular}

Numbers indicate the maximum number of neurons labeled on one side of the animal by the representative Gr-GAL4 driver for each gene.

${ }^{a}$ Number of lines with expression consistent with the representative line/number of independent lines analyzed. For example, in the case of Gr2a, eight independent lines were analyzed; in seven of them, two was the maximum number of cells observed in one $\mathrm{T} 0$ and in one D0. The line with the most penetrant expression was selected as the representative line. The one line that did not have expression consistent with the other lines had no expression at all, likely because of a positional effect. In the case of Gr5a, seven of seven lines had no expression in the larva. For Gr36a, seven ofeight lines had no expression, while one line had ectopic expression in a large number of uncharacterized cells; thus, no expression is the representative expression pattern.

${ }^{b}$ Includes a line obtained from K. Scott (University of California, Berkeley, Berkeley, CA).

Includes a line obtained from H. Amrein (Texas A\&M University, College Station, TX). 
A

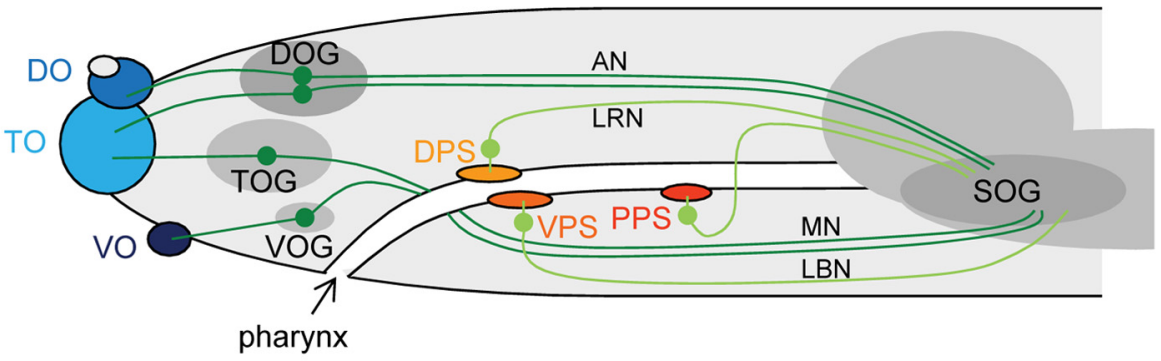

B

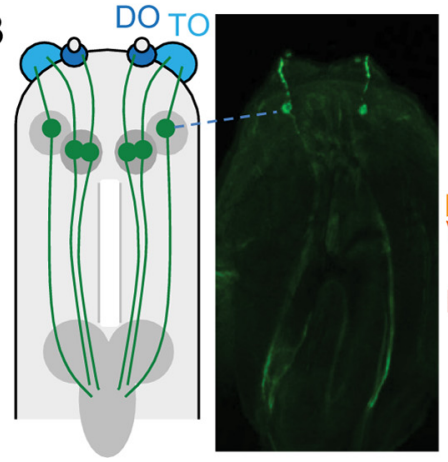

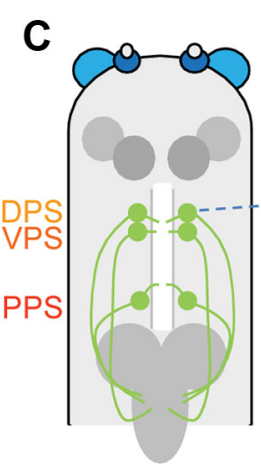

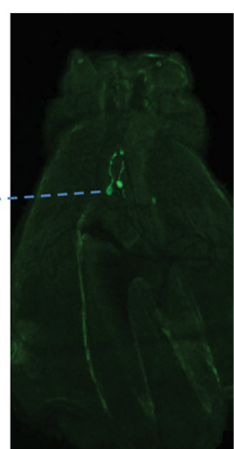

D

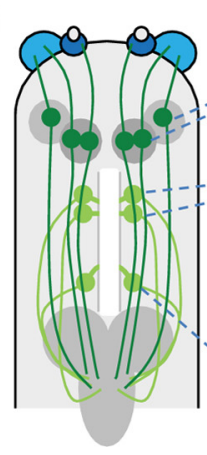

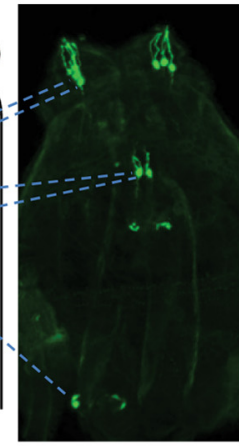

Figure 1. A, The taste system of the larval head (lateral view) [adapted from the study by Stocker (2008) with permission from Landes Bioscience/Springer]. Anterior is to the left. The D0G, TOG, and VOG are represented by gray shaded ovals and contain the cell bodies of 36-37,32, and 7 sensory neurons, respectively (Stocker, 2008). Three neurons innervating the TO have cell bodies in the DOG (TO-dorsolateral group; see Results and Fig. 2). Representative neuronal projections are depicted in green. The axons of all putative taste neurons extend to the SOG in the brain. Olfactory neurons are not represented here for simplicity. AN, Antennal nerve; LRN, labral nerve; LBN, labial nerve; $M N$, maxillary nerve. $\boldsymbol{B}-\boldsymbol{D}$, Dorsal views of the taste system in the larval head. Anterior is at the top. $\boldsymbol{B}, 0$ n the left is a schematic view of neurons (green) of the $\mathrm{TO}$ and DO. The $\mathrm{V} 0$ is not shown. On the right is an example of a specific driver, Gr59c-GAL4, which drives expression in neurons that innervate the TO. C, Left, Pharyngeal neurons (green). Right, Gr77a-GAL4 drives expression in neurons that innervate the DPS. D, Left, Neurons of the T0, D0, and pharyngeal organs are indicated in green. Right, Gr33a-GAL4 drives peripheral expression in neurons that innervate the TO as well as the pharyngeal sense organs. The VPS is located ventral to the DPS, as shown in $A$, and they are often superimposed in a dorsal or ventral view. The labeled cells between the DPS and PPS cells may be cells of the DPO (dorsal pharyngeal organ) (Gendre et al., 2004), but we could not identify them with confidence.

secondary antibodies used were goat anti-mouse and goat anti-rabbit IgG conjugated to either Alexa 568 or Alexa 488 (1:1000) (Invitrogen).

All images were collected on either a Zeiss LSM 510 laser-scanning confocal microscope or a Bio-Rad 1024 laser-scanning confocal microscope.

\section{Results}

To examine systematically the expression of the entire repertoire of Grs in the larva, we used the GAL4-UAS system. Most attempts to analyze expression of $G r$ genes by in situ hybridization have been unsuccessful, presumably due to low expression levels (Clyne et al., 2000; Dunipace et al., 2001; Scott et al., 2001; Dahanukar et al., 2007; Moon et al., 2009). The GAL4-UAS system has been a more successful approach (Brand and Perrimon, 1993; Dunipace et al., 2001; Scott et al., 2001; Chyb et al., 2003; Thorne and Amrein, 2008; Moon et al., 2009), and recently the expression of all Grs was examined in the labellum, the major taste organ of the adult head (Weiss et al., 2011). Results of this expression analysis of Gr-GAL4 lines in the labellum agreed well with the results of functional analysis (Weiss et al., 2011), supporting the utility of the approach.

The Gr-GAL4 transgenes we used are essentially those used in the study by Weiss et al. (2011), with minor differences in the number of lines examined for certain $G r$ genes and in the selection of representative lines for more detailed analysis. Collectively, the expression patterns of 67 Gr-GAL4 drivers were examined. (One driver, Gr23a-GAL4, represents two Grs, Gr23a.a and Gr23a.b, which are encoded by alternative spliceforms that share a common 5 ' region.) For some individual $G r$ GAL4 drivers, as many as 23 independent lines were examined in an effort to determine accurately the expression pattern of each gene (Table 1). For certain previously published Gr transgenes, a single line was analyzed (Dunipace et al., 2001; Scott et al., 2001; Weiss et al., 2011). In total, we analyzed 507 lines for the 67 Gr-GAL4 drivers, a mean of 7.6 lines/driver. We examined thirdinstar animals that were heterozygous for the Gr-GAL4 driver and that contained two copies of the UAS- $m C D 8$-GFP reporter.

For each $\mathrm{Gr}$, we selected one representative $G r-G A L 4$ line on the basis of a quantitative analysis. To select a representative line for a particular Gr, at least 10 animals were scored for every line established for that Gr (i.e., a total of $>5070$ animals across all drivers). The mean number of labeled neurons in each line was calculated as a measure of expression pattern. For each Gr, one expression pattern was observed among the majority of lines and was defined as the representative pattern. Among lines showing the representative pattern for a Gr, one line that showed the most consistent and penetrant expression was selected as the representative line (Table 1; see also Materials and Methods).

\section{Taste organs of the larval head express 39 Gr-GAL4 drivers}

Of the 67 Gr-GAL4 drivers, 39 showed expression in the peripheral taste organs of the head (Table 1). Among the major cephalic taste organs, we focused on the TO, DO, DPS, VPS, and PPS; expression in the $\mathrm{VO}$ was difficult to discern due to a lack of easily distinguishable features or markers.

The 39 Gr-GAL4 drivers fell into three categories. The first category contains 15 drivers that show expression in neurons innervating the sensory organs on the tip of the head (TO and/or DO), but not in the pharyngeal sense organs (DPS, VPS, or PPS) (Fig. $1 B$, Table 1). The second category consists of 11 drivers that show expression in the pharyngeal sense organs with no expres- 
A
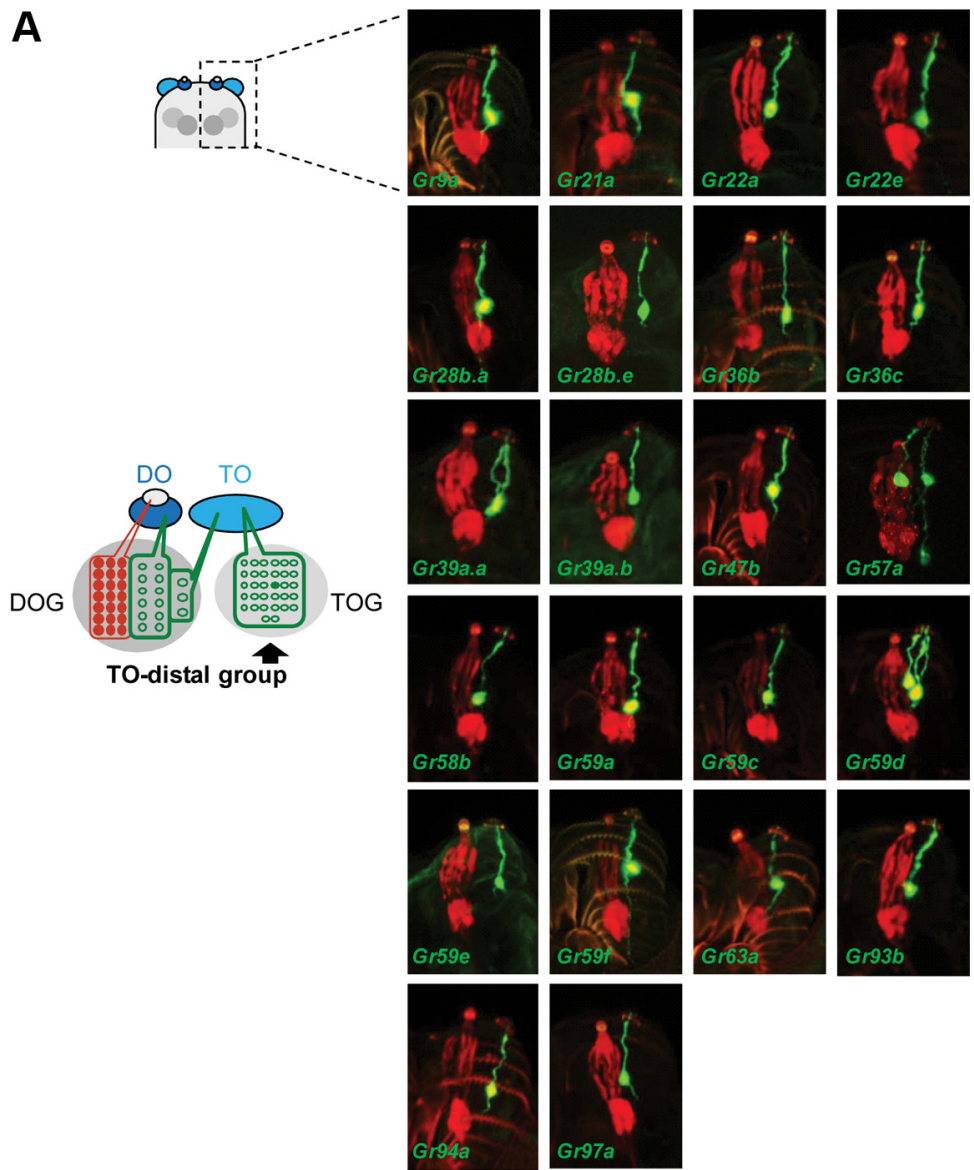

B

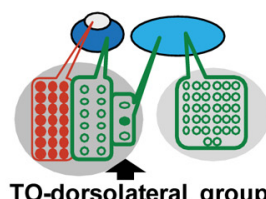

C
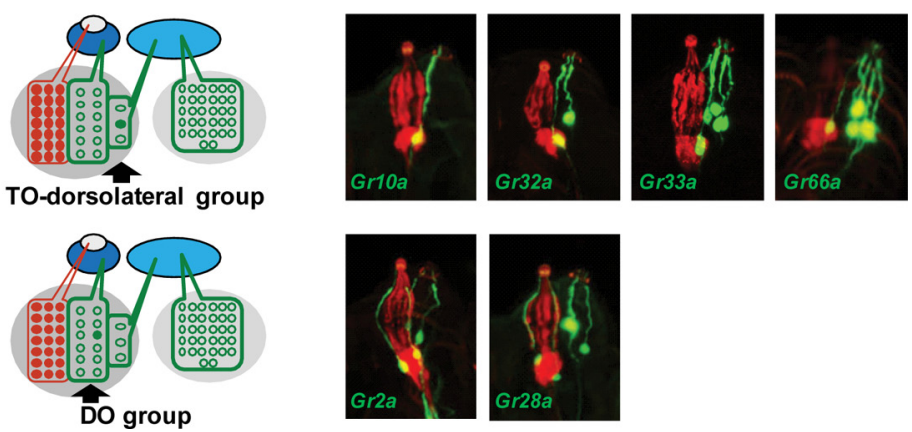

Figure 2. Division of Gr-GAL4 drivers that show expression in T0- and/or D0-innervating neurons into three classes: the T0-distal, T0-dorsolateral, and D0 classes. $\boldsymbol{A}$, The small diagram (above, left) shows a larval head, with magnified representations of the TO, DO, TOG, and DOG. The large diagram (below, left) depicts the cells of the TOG and DOG. The TOG contains the cell bodies of 32 sensory neurons that innervate the T0 (the T0-distal group of neurons) (Stocker, 2008). The photographs at the right show the 22 Gr-GAL4 drivers of the T0-distal class. Orco-RFP was used as an olfactory neuronspecific marker to indicate the position of the DOG; all 21 olfactory neurons of the DOG innervate the dome sensillum. Gr39a.b-GAL4 does not exhibit full penetrance; the photograph shows only one labeled neuron, but in many cases two neurons are labeled. $\boldsymbol{B}$, Three neurons from the DOG innervate the TO (the TO-dorsolateral group). Four Gr-GAL4 drivers fell into the T0-dorsolateral class. Gr32a-GAL4, Gr33a-GAL4, and Gr66a-GAL4 also showed expression in the T0-distal neurons. In the case of Gr33a-GAL4 and Gr66a-GAL4, two neurons (B1 and B2 in Fig. 4) are labeled in the T0-dorsolateral group but are very close to each other, making them difficult to distinguish in a two-dimensional photographic representation. $\boldsymbol{C}$, Twelve putative taste neurons from the DOG innervate the peripheral sensilla of the DO (the DO group). Two Gr-GAL4 drivers fell into the DO class. Each also had expression in T0-distal neurons.

sion in the TO or DO (Fig. 1C, Table 1). The third category includes 13 drivers that express in both the TO/DO and the pharyngeal sense organs (Fig. 1D, Table 1).

All of the 15 drivers that express in neurons innervating the $\mathrm{TO} / \mathrm{DO}$, but not in the pharyngeal organs, express in only one TO neuron. All drivers that express in at least two neurons of the
TO/DO also show expression in the pharyngeal sense organs (Table 1 ).

We note that, in addition to the 39 drivers that express in the cephalic chemosensory organs, drivers representing Gr28b.b, Gr28b.c, Gr28b.d, and Gr89a showed larval labeling, but elsewhere: we observed projections from the body to the ventral ganglion (data not shown). Gr2a-, Gr28a-, and Gr33a-GAL4 also labeled projections from the body in addition to their expression in the head taste organs. Gr28b.d also expressed in head cells that we could not identify with confidence. Finally, we were interested to observe that of the 39 drivers that express in the cephalic chemosensory organs, 22 were recently found to be expressed in the labellum, the major organ of the adult head (Weiss et al., 2011), and at least four, Gr32a-, Gr33a-, Gr39a-, and Gr68a-GAL4, represent genes associated with courtship behavior (Bray and Amrein, 2003; Miyamoto and Amrein, 2008; Moon et al., 2009; Wang et al., 2011; Watanabe et al., 2011).

\section{Classification of $\mathrm{Gr}$ expression into TO-distal, TO-dorsolateral, and DO classes}

The 28 Gr-GAL4 drivers expressed in neurons of the TO/DO (i.e., those of the first and third categories above; Fig. $1 B, D$ ) fell into three classes. These classes were distinguished based on the positions of the labeled cell bodies and the organs that they innervate on the tip of the head, regardless of whether they label pharyngeal organs. To classify the Gr genes, the representative Gr-GAL4 driver for each $G r$ was crossed into an Orco-RFP-expressing line. Orco is a cation channel and chaperone required for odorant receptor (Or) function (Benton et al., 2006; Sato et al., 2008; Wicher et al., 2008). Orco is expressed in all 21 larval ORNs (Larsson et al., 2004) and thus marks the position of the DOG. Using Orco-RFP as a marker for the DOG allowed us to distinguish three partially overlapping classes of $G r$ drivers in the larval head: the TO-distal class, expressed in neurons with cell bodies in the TOG that innervate the TO; the TOdorsolateral class, which shows expression in neurons with cell bodies in the DOG that innervate the TO; and the DO class, which shows expression in neurons with cell bodies in the DOG that innervate the DO (Fig. 2).

The TO-distal class contains 22 Gr-GAL4s (Fig. $2 A$ ); the TOdorsolateral class contains 4 Gr-GAL4s (Fig. $2 B$ ); the DO class contains 2 Gr-GAL4s (Fig. 2C, Table 1). With the exception of Gr10a-GAL4 in the TO-dorsolateral class, the drivers of the TOdorsolateral and the DO classes also showed expression in neu- 
rons that innervate the TO from the TOG (Fig. $2 B, C$ ). We note that penetrance of the Gr28a-GAL4 driver was highly variable, but it showed somewhat more GFPexpressing neurons than previously reported (Thorne and Amrein, 2008), as well as expression in the DO neurons ( Table 1, Fig. 2C).

\section{A receptor-to-neuron map of the larval taste system}

Of the $28 \mathrm{Gr}$-GAL4 drivers expressed in neurons of the TO-distal, TO-dorsolateral, and DO groups, how many are coexpressed in individual neurons, and in how many neurons are they coexpressed? We performed a coexpression analysis designed to address fundamental questions regarding the functional organization of the larval taste system and its molecular and cellular complexity. The analysis is illustrated in Figure 3, and it has produced the receptorto-neuron map shown in Figure 4. As described below, the analysis has identified 10 neurons, which we have named A1, A2, B1, B2, and C1-C6.

To identify coexpression, we systematically examined $G r$-Gal4 lines in pairwise combinations. Representative Gr-GAL4 lines for two receptors were crossed, and progeny that carried one copy of each $G r$ GAL4 driver and two copies of UAS$m C D 8-G F P$ were scored. When progeny containing both drivers contained the same number of labeled neurons as animals containing one copy of either parental driver alone, we inferred that the neurons labeled by one driver either overlapped precisely with the neurons labeled by the other driver or were a subset of them. When the double-driver progeny contained a greater number of neurons than animals containing either driver alone, the simplest interpretation is that some or all neurons labeled by one driver are distinct from the neurons labeled by the other.

Gr33a-GAL4 and Gr66a-GAL4 drive expression in the greatest number of TO/DO neurons. Each driver labels four TO-distal neurons and two TO-dorsolateral neurons (Table 1, Fig. 2 B; not all neurons can be observed clearly in a single, two-dimensional photographic representation). When Gr33aGAL4 was combined with Gr66a-GAL4, the number of neurons expressing GFP was unchanged, showing that Gr33a-GAL4 and Gr66a-GAL4 coexpress in the same neurons of the larval head sensory organs (Fig. $3 A$, top left). This finding is consistent with their coexpression in the adult labellum, and both genes are required in adults for the response to bitter compounds (Moon et al., 2009; Weiss et al., 2011).
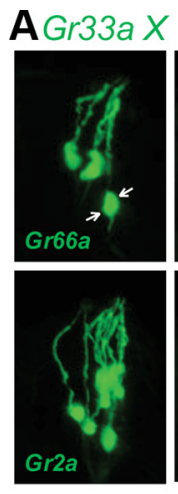

BGr21aX
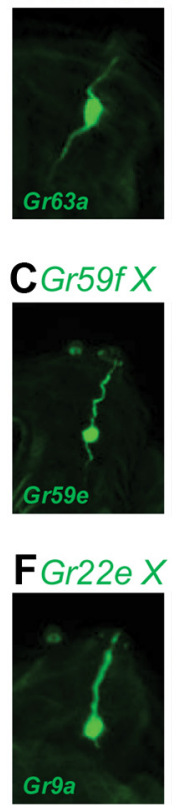
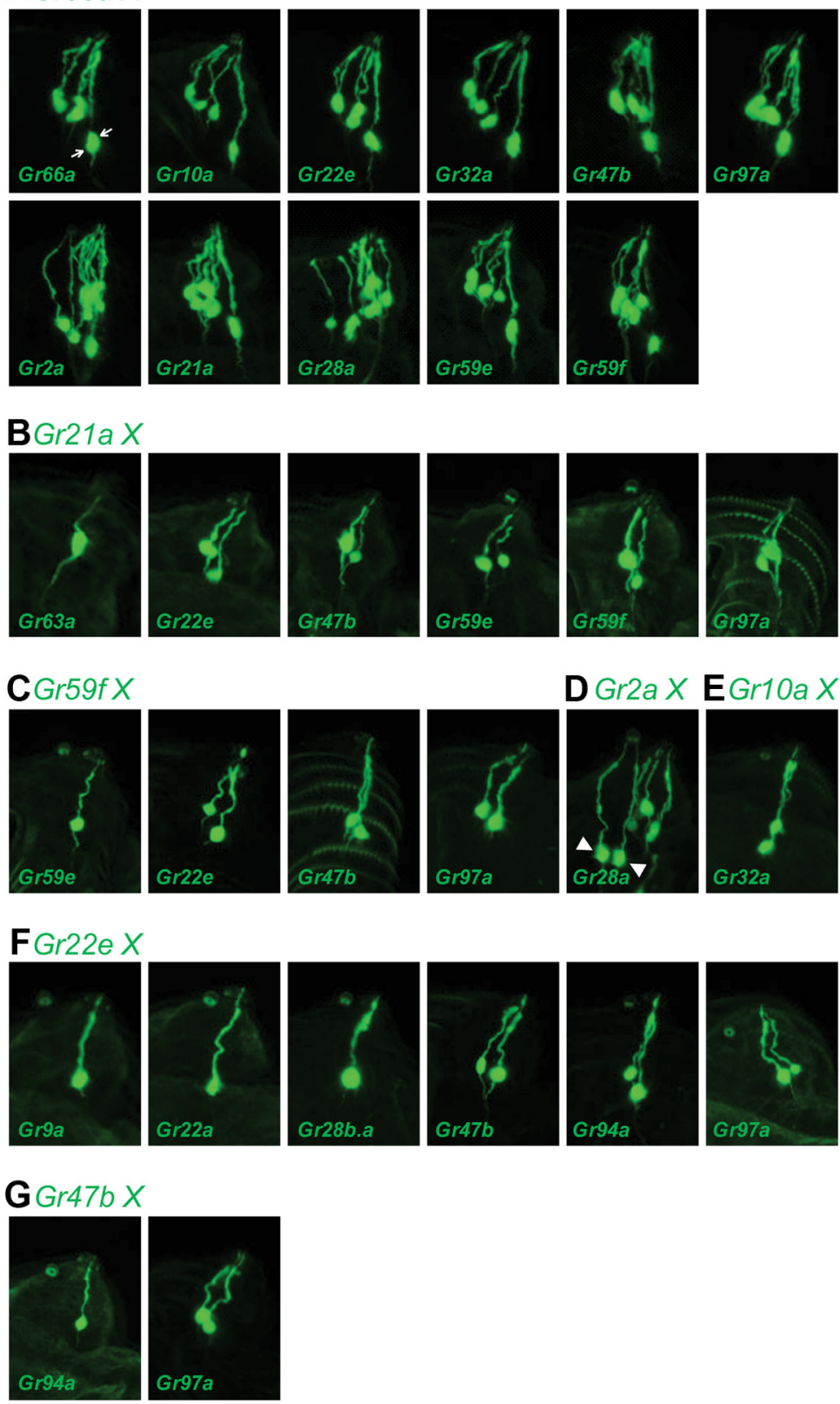

Figure 3. Determination of coexpression by double-driver analysis. The average number of neurons expressing GFP was determined from at least 10 animals of each genotype. A, Top row, When Gr33a-GAL4 was combined with Gr66a-, Gr10a-, Gr22e-, Gr32a-, Gr47b-, or Gr97a-GAL4, the number of labeled cells was the same as for Gr33a-GAL4 alone (Fig. 2B), indicating that the neurons expressed in these lines are coincident with, or a subset of, the neurons expressed by Gr33a-GAL4. Five labeled cells can be observed easily, and in each case a sixth cell body and dendrite could be seen in another focal plane; two cell bodies at the bottom right (indicated by arrows in the first image; these cells are referred to as B1 and B2 in Fig. 4) are very close to each other. Bottom row, When Gr33a-GAL4 was combined with Gr2a-, Gr21a-, Gr28a-, Gr59e-, or Gr59f-GAL4, a larger number of neurons was observed, indicating that these five drivers are expressed in at least some cells distinct from the Gr33a-GAL4-expressing neurons. B, Gr21a-GAL4 and Gr63a-GAL4 are coexpressed in a neuron that does not express several other tested Gr-GAL4 drivers. C, Gr59e-GAL4 and Gr59f-GAL4 are expressed in the same T0-distal neuron, in a neuron that does not coexpress several other tested drivers. D, Gr2a-GAL4 and Gr28a-GAL4 are coexpressed in two neurons of the D0 group (arrowheads). There is also labeling of some TO neurons. $\boldsymbol{E}$, When Gr10a-GAL4 and Gr32a-GAL4 were combined, the expression appeared identical with that of Gr32a-GAL4 alone (Fig. 2B) (i.e., one T0-dorsolateral neuron and one T0-distal neuron are labeled). F, Gr22e-GAL4 is coexpressed in a T0-distal neuron with Gr9a-GAL4, Gr22a-GAL4, and Gr28b.a-GAL4 but is not coexpressed with Gr47b-GAL4, Gr94a-GAL4, or Gr97a-GAL4. Gr22e-GAL4 was also coexpressed with drivers representing Gr28b.e, Gr32a, Gr36b, Gr36c, Gr58b, Gr59a, Gr59c, Gr93b (data not shown). G, Gr47b-GAL4 and Gr94a-GAL4 are coexpressed, in a neuron distinct from that expressing Gr97a-GAL4.

Gr33a-GAL4 was then crossed to Gr10a-, Gr22e-, Gr32a-, Gr39a.a-, Gr47b-, or Gr97a-GAL4, all of which label a smaller number of neurons than Gr33a-GAL4. The progeny showed labeling of the same number of neurons as Gr33a-GAL4, suggesting that these six 


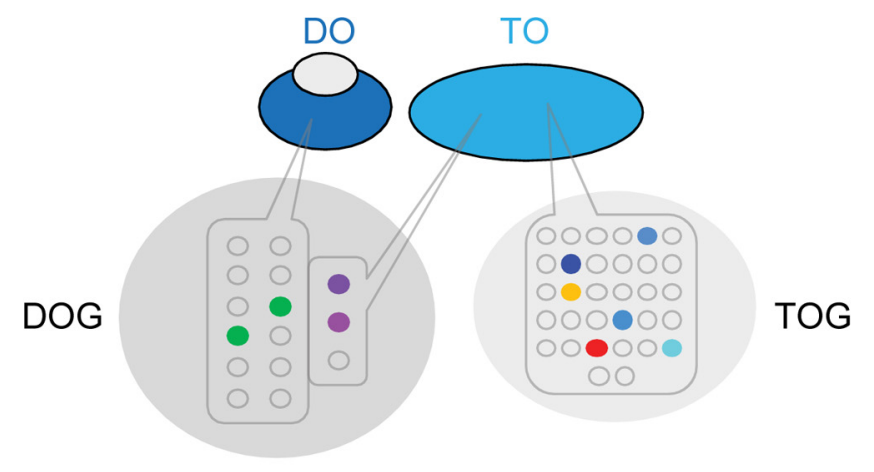

DO group TO-dorsolateral group TO-distal group

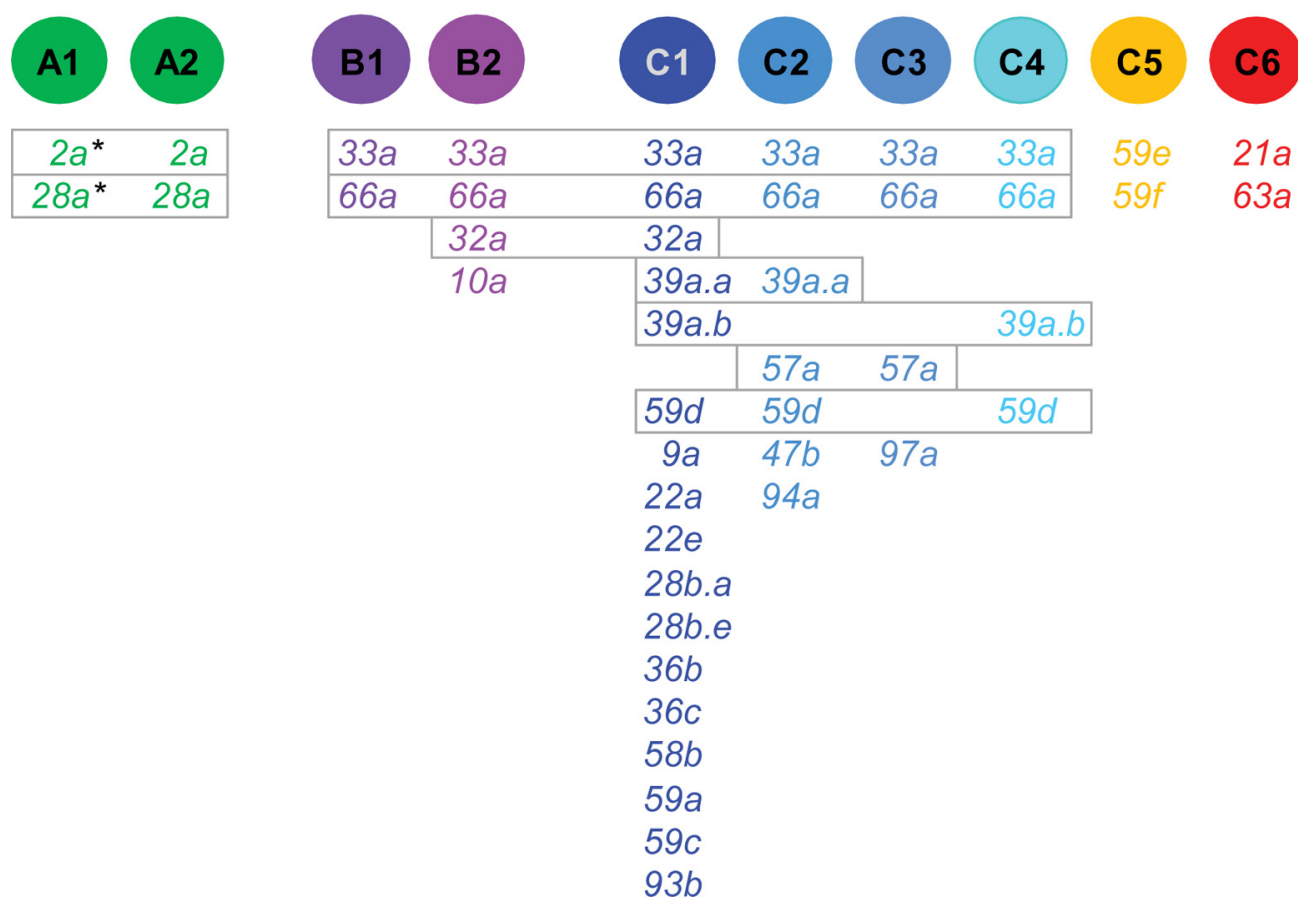

Figure 4. A receptor-to-neuron map. Coexpressed Gr-GAL4 drivers are listed under each neuron, and receptors that map to multiple neurons are boxed in gray. The asterisk (*) indicates that Gr2a-GAL4 and Gr28a-GAL4 are also expressed in up to three T0-distal neurons, but we were unable to identify them with confidence; although penetrance of expression in the D0 group was high, it was lower in T0-distal neurons. Positions of cell bodies in the diagram of the DOG and TOG are arbitrary.

receptors are expressed in a subset of Gr33a-expressing neurons (Fig. 3A, top row) (data not shown).

By contrast, when Gr2a-, Gr21a-, Gr28a-, Gr59e-, Gr59f-, and Gr63a-GAL4 were combined with Gr33a-GAL4, the number of labeled neurons was increased, showing that some or all of the cells in which these six drivers are expressed are distinct from the Gr33a-GAL4-expressing neurons (Fig. 3A, bottom row) (data not shown). Of the $28 \mathrm{Gr}$-GAL4 drivers expressed in neurons of the TO/DO, these were the only six drivers that labeled an increased number of neurons when combined with Gr33a-GAL4. We considered each of these six drivers in turn.

Gr21a-GAL4 and Gr63a-GAL4 are coexpressed in one larval neuron (Fig. $3 B$ ), as has been shown previously; they are also coexpressed in the adult. Coexpression in an in vivo expression system was sufficient to confer a response to $\mathrm{CO}_{2}$ (Jones et al., 2007; Kwon et al., 2007). We performed a series of crosses with additional drivers, none of which was coexpressed with Gr21a-GAL4 and Gr63a-GAL4 (Fig. 3B) (data not shown). This direct evidence, together with infer- ence from other crosses described below, support the conclusion that this neuron expresses only Gr21a and Gr63a. We have termed the neuron C6 (Fig. 4).

Gr59e-GAL4 and Gr59f-GAL4 are also coexpressed, in one TO-distal neuron (Fig. $3 C$ ). This neuron was confirmed directly to be distinct from the TO-distal neuron that coexpresses Gr21aGAL4 and Gr63a-GAL4 (Fig. 3B), as well as from neurons expressing Gr22e-, Gr47b-, Gr97a- (Fig. 3B) and Gr33a-GAL4 (Fig. $3 A$ ). We have denoted this neuron C5 (Fig. 4).

Gr2a-GAL4 and Gr28a-GAL4 express in two neurons of the DO group (Table 1, Fig. $2 C$ ), and their coexpression in DO neurons was demonstrated by crossing the two lines (Fig. $3 D$, arrowheads). These DO neurons are termed A1 and A2 (Fig. 4). Gr2a-GAL4 and Gr28a-GAL4 are the only drivers expressed in DO neurons. We note that Gr2a-GAL4 and Gr28a-GAL4 are also expressed, but appear not to be coexpressed, in TO-distal neurons; however, expression was variable in TO-distal neurons, it is not depicted in Figure 4, and definitive mapping will require further 

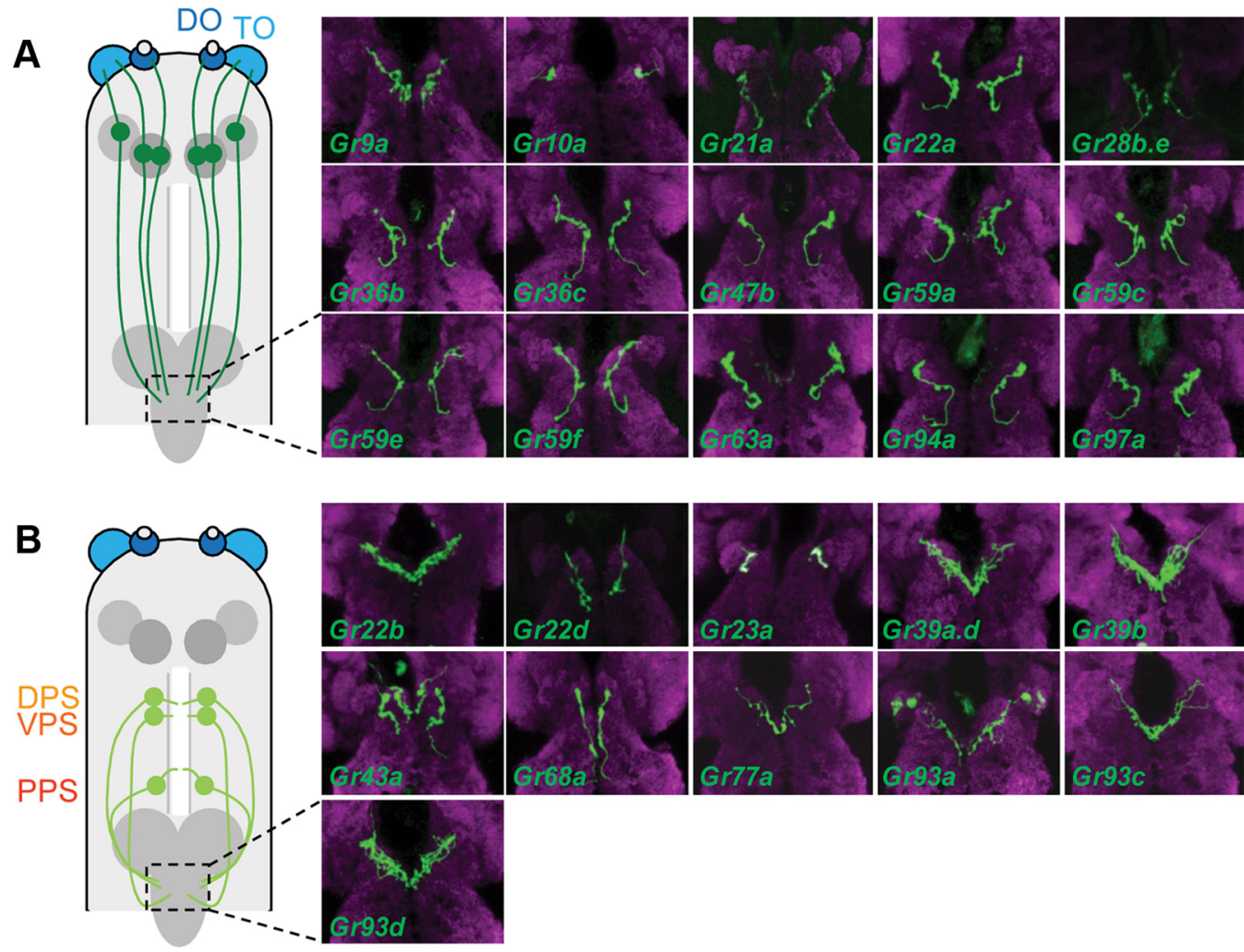

\section{C}
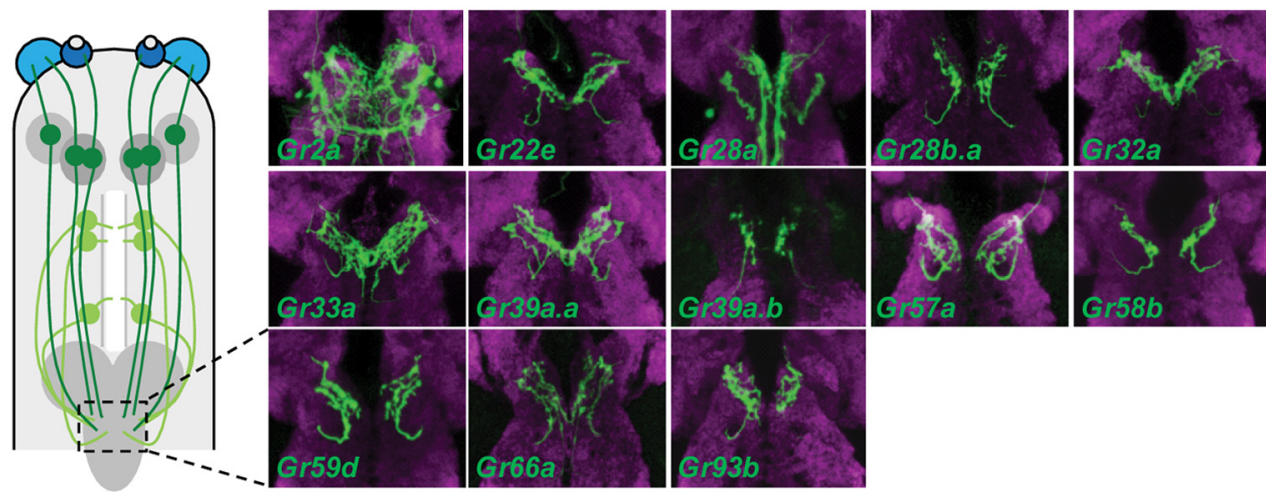

Figure 5. Projection patterns in the $S O G$ of the brain, shown for each of the $39 \mathrm{Gr}$-GAL4s expressed in head taste organs. A, Gr-GAL4 drivers that express exclusively in neurons that innervate the TO and/or DO B, Gr-GAL4 drivers that express exclusively in neurons that innervate the pharyngeal sense organs. C, Gr-GAL4 drivers that express both in TO/DO neurons and pharyngeal neurons.

analysis. Gr28a has previously been found to be expressed in various adult and larval sensory organs (Thorne and Amrein, 2008).

We analyzed in more detail the TO-dorsolateral neurons, two of which are labeled by Gr33-GAL4 and Gr66a-GAL4; they are closely adjacent and are often superimposed in dorsal views (Fig. 2B). Gr10a-GAL4 and Gr32a-GAL4 are each expressed in one of these neurons; Gr32a-GAL4 is also expressed in one TO-distal neuron (Fig. 2 B). When the Gr10a-GAL4 and Gr32a-GAL4 drivers were combined, expression was observed in two cells (Fig. $3 E$ ) and appeared identical to that of Gr32a-GAL4 alone (Fig. $2 B$ ). These results indicate that Gr10a-GAL4 and Gr32a-GAL4 are coexpressed in one TO-dorsolateral neuron, termed B2, along with Gr33a and Gr66a (Fig. 4).

Next, we examined in more detail the TO-distal neurons. Many of the Gr-GAL4 drivers are expressed in a single TO-distal neuron (Fig. 2A). We found that 12 of them, including Gr9a-, Gr22a-, and Gr28b.a-GAL4, when combined with Gr22e-GAL4, showed labeling of a single neuron, indicating that all of these drivers are expressed in a single neuron, $\mathrm{C} 1$ (Fig. $3 F$ ) (data not shown). By contrast, Gr47b-, Gr94a-, and Gr97a-GAL4, combined with Gr22e-GAL4, labeled two neurons (Fig. 3F). Additional crosses showed that Gr47b- and Gr94a-GAL4 are coexpressed in a neuron, C2, distinct from that expressing Gr97aGAL4, C3 (Fig. 3G). Using Gr22e-GAL4, Gr47b-GAL4, and Gr97a-GAL4 as markers, we performed similar double-driver experiments with Gr39a.a, Gr39a.b, Gr57a, and Gr59d (data not shown), which defined a fourth TO-distal neuron, C4 (Fig. 4).

\section{Correlations between receptor expression and central projection patterns}

We examined projection patterns in the brain of all 39 larval Gr-GAL4 lines that drive expression in the taste organs of the head. We compared the projections of three categories of drivers: the 15 drivers that show expression in the TO/DO but not in the pharyngeal organs (Figs. $1 B, 5 A$ ); the 11 drivers that show expression in the pharyngeal sense organs but not the TO/DO (Figs. $1 C$, 
$5 B$ ); and the 13 drivers that express in both the TO/DO and the pharyngeal sense organs (Figs. $1 D, 5 C$ ).

With a few exceptions, patterns within a category were more similar than patterns between categories. Among the patterns of the TO/DO category, most are similar, consistent with the conclusion from the double-driver analysis that many drivers are coexpressed in the same cell, C1. A distinct pattern is shown by Gr10-GAL4, which is the only driver in this category expressed in the TO-dorsolateral neurons, in $\mathrm{B} 2$. The neurons in this group project to the brain via the antennal nerve, whereas the TO-distal neurons such as $\mathrm{C} 1$ project via the maxillary nerve. We note that Gr21-GAL4 and Gr63a-GAL4, coexpressed in C6, also appear similar to each other and distinct from the others. This finding is consistent with the earlier finding that the Gr21a-GAL4 projection was distinct from that of others (Colomb et al., 2007).

Many patterns of the pharyngeal-only class show similarities; for example, most of these afferents can be seen to extend to, or very near, the midline. There are some differences; for example, Gr68a-GAL4 shows a distinct pattern from the others; it is also the only driver in this category that is expressed in the VPS (Table 1). Overall, however, a comparison of all members of the TO/DO and pharyngeal-only categories suggests that sensory information collected from different regions (i.e., the tip of the head vs the pharynx) is processed in different subregions of the SOG, consistent with previous studies performed with limited numbers of Gr-GAL4 drivers (Colomb et al., 2007).

Gr-GAL4 drivers with expression in both the TO/DO and pharyngeal sense organs showed more complex brain projection patterns (Fig. $5 C$ ). In a number of cases, the projections can be interpreted as a composite of the projection patterns observed in the TO-specific and pharyngeal-specific categories (Fig. $5 A, B$ ).

\section{Discussion}

\section{A comprehensive view of $G r$ expression in larvae}

Of the $67 \mathrm{Gr}$-GAL4 transgenes, 43 showed expression in the larva, of which 39 were expressed in the major taste organs of the head. The $39 \mathrm{Gr}$-GAL4 drivers are expressed in combinatorial fashion (Table 1, Fig. 4). Individual Gr-GAL4 drivers are expressed in up to 12 cells, in the case of Gr33a- and Gr66a-GAL4; approximately one-half, however, are expressed in only one cell.

We acknowledge that for some Gr-GAL4 drivers the observed pattern of expression may not be identical with that of the endogenous $G r$ gene. It was precisely with this concern in mind that we analyzed a mean of 7.6 independent lines for each of the $67 \mathrm{Gr}$ drivers, and that we established a rigorous, quantitative protocol for identifying a representative line for each gene. In the absence of an effective in situ hybridization protocol, the approach used here seemed likely to be the most informative in providing a comprehensive systems-level view of larval taste reception.

\section{Features of the receptor-to-neuron map}

The Gr receptor-to-neuron map of the dorsal and terminal organs identified 10 neurons. Two neurons have cell bodies in the DOG and innervate the DO, two have cell bodies in the DOG and innervate the TO, and six have cell bodies in the TOG and innervate the TO.

We mapped 28 receptors to these 10 neurons. All of these neurons express at least two Gr-GAL4 drivers. Two receptors, Gr21a and Gr63a, are coreceptors for $\mathrm{CO}_{2}$; neither is sufficient to confer chemosensory function alone (Faucher et al., 2006; Jones et al., 2007; Kwon et al., 2007). It is conceivable that many other Grs may also require a coreceptor, which may explain the lack of neurons expressing a single Gr-GAL4. The number of receptors per neuron ranges up to 17 , in the case of $\mathrm{C} 1$. This number is comparable with the maximum number of Gr-GAL4s observed in a labellar neuron (29), and much greater than the number of Ors observed in individual neurons of either the larval or adult olfactory system (Couto et al., 2005; Fishilevich and Vosshall, 2005; Goldman et al., 2005; Kreher et al., 2005).

Among the 10 identified cells, individual Gr-GAL4 drivers are expressed in as few as one cell and as many as six cells. Most of the drivers are expressed in only one of these 10 cells. The drivers expressed in six cells, Gr33a-GAL4 and Gr66a-GAL4, are expressed in all bitter neurons of the adult labellum. We note that Gr33a-GAL4 and Gr66a-GAL4 are the only drivers expressed in $\mathrm{B} 1$, arguing against the possibility that both of these receptors function exclusively as chaperones or as coreceptors that require another $\mathrm{Gr}$ for ligand specificity.

There is little cellular redundancy. Only two neurons, A1 and A2, express the same complement of receptors. All other neurons contain a unique subset of the $\mathrm{Gr}$ repertoire. In this respect, the larval taste system differs from the adult taste system (Weiss et al., 2011) but is similar to the larval olfactory system, which also contains little if any cellular redundancy (Fishilevich et al., 2005; Kreher et al., 2005).

Analysis of the central projections of all $39 \mathrm{Gr}$-GAL4 drivers provided evidence for a systematic difference among projection patterns between TO/DO neurons and pharyngeal neurons (Fig. 5 , compare $A, B)$. These results support the conclusion that sensory information collected from the tip of the head is processed in different regions of the SOG than information collected in the pharynx, i.e., that evaluation of a potential food source before ingestion and the testing of food quality during ingestion are functionally partitioned in the brain. Similar inferences were drawn in an elegant study of a limited number of Gr-GAL4 transgenes (Colomb et al., 2007).

\section{Sugar receptors}

Conspicuously absent from the list of Gr-GAL4 drivers expressed in the larval taste system are those representing the eight members of the sugar receptor subfamily (Gr5a, Gr61a, Gr64a-f) (Robertson et al., 2003). The founding member of this family, Gr5a, mediates response to the sugar trehalose (Dahanukar et al., 2001), and two other members of the subfamily have been shown to encode sugar receptors as well (Dahanukar et al., 2007; Slone et al., 2007; Jiao et al., 2008). We did not observe GFP expression for these genes in cells of the taste organs or in neural fibers in the brain or ventral ganglion. Most of these Gr-GAL4 transgenes drive expression in the adult (Dahanukar et al., 2007) (A. Dahanukar, J. Y. Kwon, L. A. Weiss, F. Ling, and J. R. Carlson, unpublished results), but we acknowledge that these transgenes may not faithfully reflect expression in the larva.

Given that Drosophila larvae respond to sugars (Miyakawa, 1982; Schipanski et al., 2008), as do larvae of other insect species (Dethier and Kuch, 1971; Glendinning et al., 2000; Schoonhoven and van Loon, 2002), how do they detect them without members of the sugar receptor subfamily? Other Grs, including the recently identified fructose receptor Gr43a (Sato et al., 2011), may underlie sugar detection in the larva. We note that Gr59e-GAL4 and Gr59f-GAL4 are coexpressed in a cell that does not express the bitter cell markers Gr33a-GAL4 or Gr66a-GAL4. Sugar reception may also be mediated by other kinds of receptors, such as those of the TRPA family (Xu et al., 2008). 


\section{Bitter receptors}

In adult Drosophila, Gr33a-GAL4 and Gr66a-GAL4 are coexpressed with other Gr-GAL4s in bitter neurons; the simplest interpretation of expression and functional analysis is that multiple bitter receptors are coexpressed (Thorne et al., 2004; Wang et al., 2004; Lee et al., 2009; Moon et al., 2009; Weiss et al., 2011).

In the larva, we found that most larval Gr-GAL4s are coexpressed with Gr33a- and Gr66a-GAL4, suggesting the possibility that most larval Grs act in bitter response. We note that, of the 17 Gr-GAL4s coexpressed in the C1 neuron, 15 are coexpressed in a bitter neuron of the labellum (Weiss et al., 2011). We also establish that there are distinct molecular classes of Gr33a-GAL4, Gr66a-GAL4-expressing neurons. The simplest interpretation of these results is that there are distinct bitter-sensing neurons in larvae.

Larvae must determine whether to accept or reject a food source (Tompkins, 1979; Schoonhoven and van Loon, 2002), and in principle this determination could be made by a simple binary decision-making circuit. However, the existence of six Gr33aGAL4, Gr66a-GAL4-expressing neurons expressing distinct subsets of $G r-G A L 4$ s suggests a greater level of complexity in the processing of gustatory information. One possibility is that $\mathrm{C} 1$, which expresses the largest subset of drivers among the TO/DO neurons, may activate an aversive behavior in response to many of the bitter compounds that the larva encounters, while C2, C3, $\mathrm{C} 4$, or B2 either potentiates the response or activates a different motor program in response to chemical cues of particular biological significance or exceptional toxicity. The existence of heterogenous bitter-sensing cells, some more specialized than others, is a common theme in insect larvae (van Loon and Schoonhoven, 1999; Glendinning et al., 2002; Marion-Poll and Descoins, 2002; Schoonhoven and van Loon, 2002). In particular, many species contain a taste cell that responds physiologically to many aversive compounds and whose activity deters feeding. $\mathrm{C} 1$ could be such a cell, and its coexpression of many receptors may provide the molecular basis of a broad response spectrum.

It is striking that the number of TO/DO neurons that express Gr-GAL4s is small compared with the total number of TO/DO neurons. We mapped Gr-GAL4 expression to only 10 cells in the TO/DO (although Gr2a-GAL4 and Gr28a-GAL4 were each expressed in two TO neurons that we were unable to map). The DOG and TOG contain 36-37 and 32 sensory neurons, respectively, among which 21 in the DOG are olfactory (Stocker, 2008). Thus, of the nonolfactory cells, on the order of $20-30 \%$ express Gr-GAL4 drivers. It will be interesting to determine how many of the other DOG/TOG cells express other chemoreceptor genes, such as $P p k$, Trp, or IR genes, and how many of the other neurons have mechanosensory, thermoreceptive, hygrosensory, or other sensory functions (Stocker, 2008).

The role of $G r$ genes in the larval pharyngeal organs is unknown. In adult pharyngeal sensilla, the TRPA1 channel, which detects irritating compounds, regulates proboscis extension (Kang et al., 2010). It is possible that Grs expressed in larval pharyngeal organs may also play a role in modulating feeding behavior. Of the $24 G r$-GAL4 drivers expressed in the larval pharyngeal organs, 9 are coexpressed with Gr33a-GAL4 and Gr66a-GAL4 in the TO/DO (Table 1, Fig. 4); it seems plausible that they may monitor ingested food for the presence of aversive compounds.

In summary, we have analyzed essential features of the molecular and cellular organization of a numerically simple taste system in a genetic model organism. We have defined 10 gustatory receptor neurons and have provided evidence that they express
Grs in combinatorial fashion, with most of these neurons and receptors acting in the perception of bitter compounds. The results lay a foundation for a molecular and genetic analysis of how these receptors and neurons, and the downstream circuitry, underlie a critical decision: whether to accept or reject a food source.

\section{References}

Benton R, Sachse S, Michnick SW, Vosshall LB (2006) Atypical membrane topology and heteromeric function of Drosophila odorant receptors in vivo. PLoS Biol 4:e20.

Brand AH, Perrimon N (1993) Targeted gene expression as a means of altering cell fates and generating dominant phenotypes. Development 118:401-415.

Bray S, Amrein H (2003) A putative Drosophila pheromone receptor expressed in male-specific taste neurons is required for efficient courtship. Neuron 39:1019-1029.

Chyb S, Dahanukar A, Wickens A, Carlson JR (2003) Drosophila Gr5a encodes a taste receptor tuned to trehalose. Proc Natl Acad Sci U S A 100 [Suppl 2]:14526-14530.

Clyne PJ, Warr CG, Carlson JR (2000) Candidate taste receptors in Drosophila. Science 287:1830-1834.

Colomb J, Grillenzoni N, Ramaekers A, Stocker RF (2007) Architecture of the primary taste center of Drosophila melanogaster larvae. J Comp Neurol 502:834-847.

Couto A, Alenius M, Dickson BJ (2005) Molecular, anatomical, and functional organization of the Drosophila olfactory system. Curr Biol 15:1535-1547.

Dahanukar A, Foster K, van der Goes van Naters WM, Carlson JR (2001) A $G r$ receptor is required for response to the sugar trehalose in taste neurons of Drosophila. Nat Neurosci 4:1182-1186.

Dahanukar A, Lei YT, Kwon JY, Carlson JR (2007) Two Gr genes underlie sugar reception in Drosophila. Neuron 56:503-516.

Dethier VG, Kuch JH (1971) Electrophysiological studies of gustation in lepidopterous larvae. I. Comparative sensitivity to sugars, amino acids, and glycosides. Z Vergl Physiol 72:343-363.

Dunipace L, Meister S, McNealy C, Amrein H (2001) Spatially restricted expression of candidate taste receptors in the Drosophila gustatory system. Curr Biol 11:822-835.

Faucher C, Forstreuter M, Hilker M, de Bruyne M (2006) Behavioral responses of Drosophila to biogenic levels of carbon dioxide depend on life-stage, sex and olfactory context. J Exp Biol 209:2739-2748.

Fish MP, Groth AC, Calos MP, Nusse R (2007) Creating transgenic Drosophila by microinjecting the site-specific phiC31 integrase mRNA and a transgene-containing donor plasmid. Nat Protoc 2:2325-2331.

Fishilevich E, Vosshall LB (2005) Genetic and functional subdivision of the Drosophila antennal lobe. Curr Biol 15:1548-1553.

Fishilevich E, Domingos AI, Asahina K, Naef F, Vosshall LB, Louis M (2005) Chemotaxis behavior mediated by single larval olfactory neurons in Drosophila. Curr Biol 15:2086-2096.

Gendre N, Lüer K, Friche S, Grillenzoni N, Ramaekers A, Technau GM, Stocker RF (2004) Integration of complex larval chemosensory organs into the adult nervous system of Drosophila. Development 131:83-92.

Glendinning JI, Nelson NM, Bernays EA (2000) How do inositol and glucose modulate feeding in Manduca sexta caterpillars? J Exp Biol 203:1299-1315

Glendinning JI, Davis A, Ramaswamy S (2002) Contribution of different taste cells and signaling pathways to the discrimination of "bitter" taste stimuli by an insect. J Neurosci 22:7281-7287.

Goldman AL, Van der Goes van Naters W, Lessing D, Warr CG, Carlson JR (2005) Coexpression of two functional odor receptors in one neuron. Neuron 45:661-666.

Groth AC, Fish M, Nusse R, Calos MP (2004) Construction of transgenic Drosophila by using the site-specific integrase from phage phiC31. Genetics 166:1775-1782.

Jiao Y, Moon SJ, Wang X, Ren Q, Montell C (2008) Gr64f is required in combination with other gustatory receptors for sugar detection in Drosophila. Curr Biol 18:1797-1801.

Jones WD, Cayirlioglu P, Kadow IG, Vosshall LB (2007) Two chemosensory receptors together mediate carbon dioxide detection in Drosophila. Nature 445:86-90.

Kang K, Pulver SR, Panzano VC, Chang EC, Griffith LC, Theobald DL, Gar- 
rity PA (2010) Analysis of Drosophila TRPA1 reveals an ancient origin for human chemical nociception. Nature 464:597-600.

Kreher SA, Kwon JY, Carlson JR (2005) The molecular basis of odor coding in the Drosophila larva. Neuron 46:445-456.

Kwon JY, Dahanukar A, Weiss LA, Carlson JR (2007) The molecular basis of $\mathrm{CO}_{2}$ reception in Drosophila. Proc Natl Acad Sci U S A 104:3574-3578.

Laissue PP, Reiter C, Hiesinger PR, Halter S, Fischbach KF, Stocker RF (1999) Three-dimensional reconstruction of the antennal lobe in Drosophila melanogaster. J Comp Neurol 405:543-552.

Larsson MC, Domingos AI, Jones WD, Chiappe ME, Amrein H, Vosshall LB (2004) Or83b encodes a broadly expressed odorant receptor essential for Drosophila olfaction. Neuron 43:703-714.

Lee T, Luo L (1999) Mosaic analysis with a repressible cell marker for studies of gene function in neuronal morphogenesis. Neuron 22:451-461.

Lee Y, Moon SJ, Montell C (2009) Multiple gustatory receptors required for the caffeine response in Drosophila. Proc Natl Acad Sci USA 106:4495-4500.

Lee Y, Kim SH, Montell C (2010) Avoiding DEET through insect gustatory receptors. Neuron 67:555-561.

Marella S, Fischler W, Kong P, Asgarian S, Rueckert E, Scott K (2006) Imaging taste responses in the fly brain reveals a functional map of taste category and behavior. Neuron 49:285-295.

Marion-Poll F, Descoins C (2002) Taste detection of phytoecdysteroids in larvae of Bombyx mori, Spodoptera littoralis and Ostrinia nubilalis. J Insect Physiol 48:467-476.

Miyakawa Y (1982) Behavioural evidence for the existence of sugar, salt and amino acid taste receptor cells and some of their properties in Drosophila larvae. J Insect Physiol 28:405-410.

Miyamoto T, Amrein H (2008) Suppression of male courtship by a Drosophila pheromone receptor. Nat Neurosci 11:874-876.

Moon SJ, Köttgen M, Jiao Y, Xu H, Montell C (2006) A taste receptor required for the caffeine response in vivo. Curr Biol 16:1812-1817.

Moon SJ, Lee Y, Jiao Y, Montell C (2009) A Drosophila gustatory receptor essential for aversive taste and inhibiting male-to-male courtship. Curr Biol 19:1623-1627.

Oppliger FY, Guerin PM, Vlimant M (2000) Neurophysiological and behavioural evidence for an olfactory function for the dorsal organ and a gustatory one for the terminal organ in Drosophila melanogaster larvae. J Insect Physiol 46:135-144.

Python F, Stocker RF (2002) Adult-like complexity of the larval antennal lobe of D. melanogaster despite markedly low numbers of odorant receptor neurons. J Comp Neurol 445:374-387.

Robertson HM, Warr CG, Carlson JR (2003) Molecular evolution of the insect chemoreceptor gene superfamily in Drosophila melanogaster. Proc Natl Acad Sci U S A 100 [Suppl 2]:14537-14542.

Sato K, Pellegrino M, Nakagawa T, Nakagawa T, Vosshall LB, Touhara K (2008) Insect olfactory receptors are heteromeric ligand-gated ion channels. Nature 452:1002-1006.

Sato K, Tanaka K, Touhara K (2011) Sugar-regulated cation channel formed by an insect gustatory receptor. Proc Natl Acad Sci U S A 108:11680-11685.

Schipanski A, Yarali A, Niewalda T, Gerber B (2008) Behavioral analyses of sugar processing in choice, feeding, and learning in larval Drosophila. Chem Senses 33:563-573.

Schoonhoven LM, van Loon JJA (2002) An inventory of taste in caterpillars: each species is its own key. Acta Zool Acad Sci Hung 48 [Suppl 1]:215-263.

Scott K, Brady R Jr, Cravchik A, Morozov P, Rzhetsky A, Zuker C, Axel R (2001) A chemosensory gene family encoding candidate gustatory and olfactory receptors in Drosophila. Cell 104:661-673.

Slone J, Daniels J, Amrein H (2007) Sugar receptors in Drosophila. Curr Biol 17:1809-1816.

Stocker RF (1994) The organization of the chemosensory system in Drosophila melanogaster: a review. Cell Tissue Res 275:3-26.

Stocker RF (2008) Design of the larval chemosensory system. Adv Exp Med Biol 628:69-81.

Thorne N, Amrein H (2008) Atypical expression of Drosophila gustatory receptor genes in sensory and central neurons. J Comp Neurol 506: $548-568$.

Thorne N, Chromey C, Bray S, Amrein H (2004) Taste perception and coding in Drosophila. Curr Biol 14:1065-1079.

Tompkins L (1979) Developmental analysis of two mutations affecting chemotactic behavior in Drosophila melanogaster. Dev Biol 73:174-177.

van Loon JJA, Schoonhoven LM (1999) Specialist deterrent chemoreceptors enable Pieris caterpillars to discriminate between chemically different deterrents. Entomol Exp Appl 91:29-35.

Vosshall LB, Stocker RF (2007) Molecular architecture of smell and taste in Drosophila. Annu Rev Neurosci 30:505-533.

Wagh DA, Rasse TM, Asan E, Hofbauer A, Schwenkert I, Dürrbeck H, Buchner S, Dabauvalle MC, Schmidt M, Qin G, Wichmann C, Kittel R, Sigrist SJ, Buchner E (2006) Bruchpilot, a protein with homology to ELKS/ CAST, is required for structural integrity and function of synaptic active zones in Drosophila. Neuron 49:833-844.

Wang L, Han X, Mehren J, Hiroi M, Billeter JC, Miyamoto T, Amrein H, Levine JD, Anderson DJ (2011) Hierarchical chemosensory regulation of male-male social interactions in Drosophila. Nat Neurosci 14:757-762.

Wang Z, Singhvi A, Kong P, Scott K (2004) Taste representations in the Drosophila brain. Cell 117:981-991.

Watanabe K, Toba G, Koganezawa M, Yamamoto D (2011) Gr39a, a highly diversified gustatory receptor in Drosophila, has a role in sexual behavior. Behav Genet 41:746-753.

Weiss LA, Dahanukar A, Kwon JY, Banerjee D, Carlson JR (2011) The molecular and cellular basis of bitter taste in Drosophila. Neuron 69:258-272.

Wicher D, Schäfer R, Bauernfeind R, Stensmyr MC, Heller R, Heinemann SH, Hansson BS (2008) Drosophila odorant receptors are both ligand-gated and cyclic-nucleotide-activated cation channels. Nature 452:1007-1011.

Xu J, Sornborger AT, Lee JK, Shen P (2008) Drosophila TRPA channel modulates sugar-stimulated neural excitation, avoidance and social response. Nat Neurosci 11:676-682. 